\title{
The influence of agricultural system, stand structural complexity and landscape context on foraging birds in oil palm landscapes
}

\author{
BADRUL AZHAR, ${ }^{1,2 *}$ DAVID B. LINDENMAYER, ${ }^{1}$ JEFF WOOD, ${ }^{1}$ JOERN FISCHER, ${ }^{3}$ ADRIAN MANNING, ${ }^{1}$ \\ CHRIS MCELHINNY ${ }^{1}$ \& MOHAMED ZAKARIA ${ }^{2}$ \\ ${ }^{1}$ The Fenner School of Environment and Society, ANU College of Medicine, Biology, and Environment, The Australian \\ National University, Canberra, ACT, Australia \\ ${ }^{2}$ Department of Forest Management, Faculty of Forestry, Universiti Putra Malaysia, Serdang, Selangor, Malaysia \\ ${ }^{3}$ Faculty of Sustainability, Leuphana University Lueneburg, Lueneburg, Germany
}

\begin{abstract}
Functional diversity, an important element of avian biodiversity, can be examined by quantifying foraging guild composition. Understanding the ecological processes that underpin functional diversity of birds in oil palm Elaeis guineensis landscapes is important because different foraging guilds are likely to be influenced in different ways by land use practices. We surveyed birds at 55 sites within oil palm landscapes and at 20 sites within logged peat swamp forest, recording 208 species belonging to 19 foraging guilds. Oil palm landscapes supported a lower abundance of insectivorous, granivorous and omnivorous birds than did logged peat swamp forest despite the latter being severely degraded due to intensive timber extraction. However, abundances of other groups of foraging birds, such as raptors and wetland taxa, were higher in oil palm landscapes than logged peat swamp forest. Frugivorous species were more abundant in smallholdings than plantation estates, probably because of the presence of native trees. Foraging guild diversity was explained by stand-level attributes such as stand age, vegetation cover, epiphyte persistence and canopy cover. However, each foraging guild exhibited unique responses to different oil palm management regimes and stand-level attributes. Only arboreal omnivores and terrestrial frugivores were affected by the proximity of nearby natural forest. This diversity of responses implies that the occurrence of particular avian foraging guilds may not be a suitable ecological indicator of best-practice palm oil production. Our study also suggests that multiple conservation measures will be needed in oil palm landscapes irrespective of management regimes, including: (1) the maintenance of ground layer vegetation cover; (2) the pruning of oil palm canopy to permit light penetration to the ground layer; (3) re-vegetation of parts of oil palm landscapes with native trees; and (4) retention of natural and/or secondary forest patches within the boundaries of plantations.
\end{abstract}

Keywords: foraging guilds, logging, peat swamp forest, plantation estates, smallholdings.

Bird foraging behaviour and guild structure are critically important determinants of bird species persistence in agricultural areas (Miller \& Cale 2000, Soderstrom et al. 2003, Sekercioglu et al. 2007). The diversity of foraging guilds in agricultural areas reflects the types of food that are

*Corresponding author.

Email: b_azhar@putra.upm.edu.my available (Blake 1983) and the methods of foraging employed (Simberloff \& Dayan 1991).

Oil palm Elaeis guineensis landscapes sustain a far lower diversity of birds than primary forest (Aratrakorn et al. 2006, Peh et al. 2006, Koh 2008, Danielsen et al. 2009, Sheldon et al. 2010). Compared with areas dominated by other commodities such as coffee Coffea spp. and Cacao Theobroma cacao (Greenberg et al. 1997, Reitsma 
et al. 2001, Mas \& Dietsch 2004, Estrada et al. 2006, Clough et al. 2007, Abrahamczyk et al. 2008), few studies have examined bird species richness and foraging guild diversity in oil palm landscapes. This knowledge gap needs to be addressed urgently because oil palm cultivation is likely to continue expanding, including into regions such as the Brazilian Amazon (Butler \& Laurance 2009).

Even in the case of ecological best-practice, landscapes dominated by palm-oil production are unable to replace the losses in biodiversity and ecosystem services caused by the clearance of peat swamp or lowland dipterocarp forest (Edwards et al. 2010, Wilcove \& Koh 2010). In Malaysia alone in 2011, oil palms planted in plantation estates and smallholdings covered 4271653 ha and 689200 ha, respectively (Malaysia Palm Oil Board 2011). However, accepting that commercial oil palm cultivation is now well established in southeast Asia and other tropical regions, identifying management practices that maintain biodiversity in these landscapes can improve current oil palm management regimes (e.g. conventional plantation estates and semi-traditional smallholdings).

Most scientific studies to date have emphasized the immediate negative impacts of natural forest conversion to oil palm. Few have suggested ways to improve conservation outcomes in established oil palm-dominated landscapes (Koh 2008, Najera \& Simonetti 2010a). In addition, nearly all studies investigating the ecological impacts of the palm oil industry have been limited to large plantation estates and have not considered the potential biodiversity values of smallholdings.

In this study, we sought to understand the effects of management-related plantation attributes (Donald 2004) on bird functional diversity as measured by the diversity of foraging guilds. It is important to understand foraging guilds because the diversity of guilds is strongly related to food resource availability (Sekercioglu et al. 2002, Dietsch et al. 2007, Tscharntke et al. 2008). Understanding the number of individual bird species in a guild will reflect the availability of the dominant resource supporting them in agricultural landscapes (Wong 1986, Carlo et al. 2004).

We posed several questions to shed new light on how to manage oil palm landscapes for better conservation outcomes. First, we assessed whether the total number of foraging guilds varied between different habitat types. We predicted that oil palm landscapes would support a lower number of foraging guilds than logged peat swamp forest. Secondly, we assessed whether different oil palm management regimes (plantation estates vs. smallholdings) and stand-level attributes influence the total number of foraging guilds in oil palm landscapes. We predicted that functional diversity would be more strongly related to stand-level attributes than management regimes and landscapelevel attributes. Thirdly, we assessed whether different oil palm management regimes and standlevel attributes influence foraging guilds. We predicted that different foraging guilds (each guild comprised multiple bird species) would respond uniquely to those attributes.

\section{METHODS}

\section{Study sites}

We conducted the study on the west coast of Peninsular Malaysia, in three provincial states: Perak, Selangor and Negeri Sembilan (between $4^{\circ} 29^{\prime} 09^{\prime \prime}$ $\mathrm{N}, \quad 100^{\circ} 42^{\prime} 47^{\prime \prime} \mathrm{E}$ and $2^{\circ} 29^{\prime} 00^{\prime \prime} \mathrm{N}, 101^{\circ} 56^{\prime} 35^{\prime \prime} \mathrm{E}$ ) (Fig. S1). The areas cultivated with oil palm within these states are 387 214, 135004 and 165384 ha, respectively (Malaysia Palm Oil Board 2011).

We surveyed 41 plantation estates and 14 smallholdings from January to September 2009. Cultivated areas were established between 5 and > 90 years ago, on former peat swamp forest, lowland dipterocarp forest and on areas formerly dominated by other commodity crops (e.g. Rubber Hevea brasiliensis and Coconuts Cocos nucifera). Plantation estates were managed by six conventional plantation companies, defined as companies that require major business capital and use modern equipment (e.g. mechanical harvesters) to manage oil palm production lands. These companies were the Sime Darby Plantation, United Plantation (UP), Malaysian Airports Agriculture and Horticulture, the Federal Land and Development Agency (FELDA), the Federal Land and Consolidation and Rehabilitation Authority (FELCRA) and a subsidiary company of the Selangor State Government. These estates accounted for $c .91000$ ha of planted oil palms, with individual estates ranging from 150 to 16000 ha. Oil palm plantation estates were managed intensively by a large workforce. The perimeters of plantation estates were guarded and fenced to deter harvest theft and poaching of wildlife. 
We defined smallholdings as semi-traditional cultivation areas that covered $>4$ ha and were operated by individual owners who used semitraditional farming methods (e.g. manual work and intercropping plants). Smallholdings typically supported multi-age stands where oil palm plants were intercropped with other commercial plants (e.g. banana Musa, coconut, Cassava Manihot esculenta, coffee, Pineapple Ananas comosus or indigenous fruit trees).

No large, intact primary forest exists on the west coast of the peninsula that could be used to establish control sites (Gardner et al. 2007). Undisturbed protected areas (> $10000 \mathrm{ha}$ ) were confined to the Titiwangsa Range of the peninsula, which is located further inland. These protected areas make up the majority of primary lowland rainforest in Peninsular Malaysia, and support very different environmental conditions and faunal assemblages from those found in coastal oil palm sites. For this reason, we collected comparative data in the North Selangor Peat Swamp Forest (NSPSF). The NSPSF covers c. 78000 ha of logged peat swamp forest (95\%) and lowland dipterocarp forest $(<5 \%)$. The NSPSF comprises three management units: Sungai Karang Forest Reserve (c. 36000 ha), Raja Musa Forest Reserve (c. $37000 \mathrm{ha}$ ) and Sungai Dusun Wildlife Reserve (c. 5000 ha). Commercial logging in the NSPSF, which reduced the abundance of large forest trees, ceased at least 19 years ago. To reduce the potential geographical bias (e.g. local climate and terrain), all study sites were located near to coastlines and on flat plains rather than in inland and in hill areas.

\section{Bird surveys}

To record birds in study sites, three experienced local observers (B. Azhar, A. Jambari and N. L. Ibrahim) from the Wildlife Ecological Research Unit, Faculty of Forestry, Universiti Putra Malaysia, surveyed a total of 470 transects, with 418 and 52 transects located in plantation estates and smallholdings, respectively. Because of major variations in the area planted and the difficult terrain in plantation estates, we used variable-length line transects (mean transect length \pm se in plantation: $348 \pm 11 \mathrm{~m}$, smallholding: $221 \pm 12 \mathrm{~m}$ and forest: $1139 \pm 101 \mathrm{~m})$, which is a suitably flexible method (Anderson et al. 1979). We recorded only individuals detected within $100 \mathrm{~m}$ of the transect.
The vegetation structure of oil palm sites is not as complex as in logged peat swamp forest. Most oil palm sites were similar in terms of planting distance between oil palms. In addition, most oil palm sites were characterized by straight-line harvesting paths within planting blocks (Turner \& Gillbanks 1974, Piggott 1990). Visibility in oil palm sites was usually greater $(>100 \mathrm{~m})$ than in the forest because ground vegetation was trimmed, controlled through the application of herbicides, or grazed by cattle.

In oil palm cultivation blocks we established transects only on harvesting paths. We avoided surveying along plantation roads due to disturbance by vehicles. We surveyed each transect in the oil palm landscapes once by walking slowly between 07:00 and 12:00 $\mathrm{h}$ or between 16:00 and 19:00 $\mathrm{h}$ on fine days. To account for diurnal differences in bird activity, we surveyed 295 and 123 plantation transects, 39 and 13 smallholding transects, and 65 and 23 peat swamp forest transects in the morning and late afternoon, respectively. As we recorded only a small number of bird species from each visit to logged peat swamp forest due to denser vegetation, this area was surveyed more intensively (forest transects were surveyed between one and 10 times) to maximize the number of species identified. To standardize sampling, we used a simple encounter rate (number of individuals sighted or heard per km) (Barlow et al. 2007). We assumed this encounter rate was a proxy for relative bird abundance because absolute abundance was not known (Barlow et al. 2007), and most survey techniques sample populations imperfectly (Mackenzie et al. 2005). Some workers have refrained from using sampling methods that can estimate absolute population abundance because of statistical problems (Felton et al. 2008).

We surveyed 20 peat swamp forest transects in the NSPSF either by walking abandoned logging roads, trails or by boat (moving downstream without using the engine). We classified forest transects as forest interior (both sides forested; canopy closed), open forest (both sides forested; canopy open) and forest edge (only one side forested). Non-forest species were excluded from the forest edge surveys.

We spaced all transects and study areas $\geq 500 \mathrm{~m}$ apart to assist spatial independence of bird observations. Because it was difficult to determine the exact age of cultivation blocks, we classified stand age of oil palm into seven categories ( $<6$ years; $6-10$ years; $11-15$ years; $16-20$ years; 
21-25 years; $>25$ years; mixed-age stands). These age classes contained 63, 95, 55, 63, 74, 68 and 52 transects, respectively.

We detected birds either visually or acoustically, and recorded all resident and migratory species (Jeyarajasingam \& Pearson 1999, Robson 2008). We taped unknown bird vocalizations using a digital recorder (Edirol R90 High Resolution by Roland) with an external stereomicrophone. We then confirmed vocalizations using an audio guide for the region (Scharringa 2005).

\section{Bird foraging guilds}

We assigned dietary niche and foraging methods to each bird species using published information about guild definitions from previous studies and regional bird guides (Table S1) (Wong 1986, Jeyarajasingam \& Pearson 1999, De Chenon \& Susanto 2006, Robson 2008). We defined 19 foraging guilds: (1) aerial sweeping insectivores, (2) arboreal sallying insectivores, (3) arboreal gleaning insectivores, (4) arboreal omnivores, (5) arboreal frugivores, (6) terrestrial gleaning insectivores, (7) barkprobing insectivores, (8) terrestrial sallying insectivores, (9) terrestrial omnivores, (10) terrestrial carnivores, (11) terrestrial granivores, (12) terrestrial frugivores, (13) wetland omnivores, (14) wetland carnivores, (15) wetland herbivores, (16) wetland granivores, (17) nocturnal sallying insectivores, (18) nocturnal raptors and (19) diurnal raptors. We further defined 'forest bird species' as those that occur only in continuous areas of native forest.

\section{Assessment of stand-level characteristics in oil palm landscapes}

We collected data on stand-level characteristics from 3141 plots, across the different oil palm management regimes (Table 1). For each transect, we estimated the percentage of vegetation cover on the ground, plant debris and bare ground in a $10 \times 10-\mathrm{m}$ plot (defined by oil palm plants at each corner - Turner \& Gillbanks 1974) every $50 \mathrm{~m}$. Undergrowth heights around oil palms were measured at two random locations along each transect using a tape measure. We used a GRS densitometer to determine canopy cover every $20 \mathrm{~m}$.

We determined epiphyte persistence on oil palms within each plot. Epiphytes were recorded as present if $10 \%$ of the trunk of an oil palm plant was covered and absent otherwise. We measured altitude using a handheld Global Positioning System (GPS) receiver (Garmin $60 \mathrm{CSX}$ ) at the start and end points of each transect.

We recorded the presence of cattle grazing and abundance of animal predators on each transect. Apart from field detection of cattle (e.g. encounter, footprints and dung), we questioned oil palm stakeholders about whether cattle grazing was part of the integrated management of a given area of oil palm planting. In addition, we counted both native and exotic animal predators (except birds of prey) on each transect (Table 1).

\section{Landscape metrics}

ARCGIS version 9.3 (ESRI, Redlands, CA, USA) was used to calculate four landscape metrics using a digital land-use database provided by the Malaysian Department of Agriculture. Using proximity analysis (near function: point to polygon measurement) and spatial analysis (polygon area), we computed isolation of transect mid-points from the nearest edges of continuous and fragmented forest and their respective area (Table 1). To calculate forest cover within $5 \mathrm{~km}$ of each oil palm site, we used buffering on the polygons.

\section{Statistical analysis}

To test our first prediction, we used generalized linear mixed models (GLMMs) (Schall 1991) in GENSTAT 12 (VSN International, Hemel Hempstead, UK). Models used a quasi-Poisson distribution with a log-link function. The total number of foraging guilds was the response variable. We fitted habitat type as a fixed effect (i.e. plantation, smallholding and peat swamp forest). Log-transformed transect length was included as an offset. Survey time (a.m. vs. p.m.) was fitted as a binary categorical predictor.

To test our second prediction, we used similar procedures to those described above. To avoid confounding between management regime and stand age, two separate habitat models were fitted, one fitting management regime (Model 1) and the other fitting stand age (Model 2). Models were selected by sequentially adding predictor variables to this initial model. We included 12 predictor variables as fixed effects (Table 1). After Pearson correlation, bare ground and debris cover were removed due to strong collinearity $(r=-0.91$ and $r=-0.60$, respectively). To obtain a better model fit, several 
Table 1. Overview and summary statistics of explanatory categorical and continuous variables used in the modelling process.

\begin{tabular}{|c|c|c|c|c|}
\hline Explanatory variable & Effect/description & $\begin{array}{l}\text { Type of } \\
\text { variable }\end{array}$ & Mean & se \\
\hline \multicolumn{5}{|l|}{ Agricultural system } \\
\hline Management regime & $\begin{array}{l}\text { Agricultural intensity/hunting pressure. Plantation estate (1) or } \\
\text { smallholding (2). }\end{array}$ & Factor & - & - \\
\hline \multicolumn{5}{|l|}{ Stand-level } \\
\hline Stand age & $\begin{array}{l}\text { Habitat. The age of oil palm was }<6 \text { years }(1), 6-10 \text { years }(2) \text {, } \\
11-15 \text { years }(3), 16-20 \text { years }(4), 21-25 \text { years }(5),>25 \text { years }(6) \text {, } \\
\text { or mixed-age stands }(7) \text {. }\end{array}$ & Cont. & 3.86 & 0.09 \\
\hline Elevation (m) & Habitat. Average of altitude measured above sea level. & Cont. & $\begin{array}{l}16.49^{\mathrm{a}} \\
11.01^{\mathrm{b}}\end{array}$ & $\begin{array}{l}0.64^{\mathrm{a}} \\
0.67^{\mathrm{b}}\end{array}$ \\
\hline Vegetation cover (\%) & $\begin{array}{l}\text { Habitat. Average } \% \text { of vegetation cover estimated in } \\
10 \times 10-\mathrm{m} \text { plots. }\end{array}$ & Cont. & $\begin{array}{l}50.08^{\mathrm{a}} \\
31.29^{\mathrm{b}}\end{array}$ & $\begin{array}{l}1.13^{\mathrm{a}} \\
3.16^{\mathrm{b}}\end{array}$ \\
\hline Debris or litter cover (\%) & $\begin{array}{l}\text { Habitat. Average } \% \text { of debris or litter cover estimated in } \\
10 \times 10-\mathrm{m} \text { plots. }\end{array}$ & Cont. & $\begin{array}{l}16.16^{\mathrm{a}} \\
25.41^{\mathrm{b}}\end{array}$ & $\begin{array}{l}0.45^{\mathrm{a}} \\
1.66^{\mathrm{b}}\end{array}$ \\
\hline Bare ground cover (\%) & $\begin{array}{l}\text { Habitat. Average } \% \text { of bare ground cover estimated in } \\
10 \times 10-\mathrm{m} \text { plots. }\end{array}$ & Cont. & $\begin{array}{l}33.77^{\mathrm{a}} \\
43.30^{\mathrm{b}}\end{array}$ & $\begin{array}{l}0.92^{\mathrm{a}} \\
3.16^{\mathrm{b}}\end{array}$ \\
\hline Undergrowth height $(\mathrm{m})$ & $\begin{array}{l}\text { Habitat. Average height of understorey vegetation measured in } \\
10 \times 10 \text {-m plots. }\end{array}$ & Cont. & $\begin{array}{l}39.65^{\mathrm{a}} \\
17.93^{\mathrm{b}}\end{array}$ & $\begin{array}{l}1.46^{\mathrm{a}} \\
3.51^{\mathrm{b}}\end{array}$ \\
\hline Canopy cover (\%) & $\begin{array}{l}\text { Habitat. Average \% of canopy cover measured in } \\
10 \times 10-\mathrm{m} \text { plots. }\end{array}$ & Cont. & $\begin{array}{l}74.89^{\mathrm{a}} \\
70.40^{\mathrm{b}}\end{array}$ & $\begin{array}{l}1.38^{\mathrm{a}} \\
2.22^{\mathrm{b}}\end{array}$ \\
\hline Epiphyte persistence (\%) & $\begin{array}{l}\text { Habitat. Average } \% \text { of epiphyte persistence on oil palm trunks } \\
\text { recorded in } 10 \times 10-\mathrm{m} \text { plots. }\end{array}$ & Cont. & $\begin{array}{l}72.78^{\mathrm{a}} \\
82.09^{\mathrm{b}}\end{array}$ & $\begin{array}{l}1.80^{\mathrm{a}} \\
3.85^{\mathrm{b}}\end{array}$ \\
\hline Cattle grazing & $\begin{array}{l}\text { Habitat. Binary variables: Presence }(1) \text { or absence }(0) \\
\text { of cattle grazing. }\end{array}$ & Factor & - & - \\
\hline Predation rate & $\begin{array}{l}\text { Number of feral dogs, Long-Tailed Macaque Macaca fasicularis } \\
\text { and Monitor Lizard Varanus salvator or V. bengalensis. }\end{array}$ & Cont. & $\begin{array}{l}0.78^{\mathrm{a}} \\
1.62^{\mathrm{b}}\end{array}$ & $\begin{array}{l}0.13^{\mathrm{a}} \\
0.66^{\mathrm{b}}\end{array}$ \\
\hline \multicolumn{5}{|l|}{ Landscape-level } \\
\hline Forest cover (ha) & $\begin{array}{l}\text { Population source. Cumulative area size of fragmented primary or } \\
\text { secondary forest within } 5 \mathrm{~km}\end{array}$ & Cont. & $\begin{array}{c}4203^{\mathrm{a}} \\
12971^{\mathrm{b}}\end{array}$ & $\begin{array}{l}846.20^{\mathrm{a}} \\
4173^{\mathrm{b}}\end{array}$ \\
\hline $\begin{array}{l}\text { Distance to the nearest } \\
\text { forest patch }(\mathrm{km})\end{array}$ & Population source. The shortest distance to forest patch. & Cont. & $\begin{array}{l}1.61^{\mathrm{a}} \\
1.68^{\mathrm{b}}\end{array}$ & $\begin{array}{l}0.05^{\mathrm{a}} \\
0.17^{\mathrm{b}}\end{array}$ \\
\hline $\begin{array}{l}\text { Isolation from the nearest } \\
\text { continuous forest }(\mathrm{km})\end{array}$ & Population source. The shortest distance to continuous forest. & Cont. & $\begin{array}{l}28.38^{\mathrm{a}} \\
30.95^{\mathrm{b}}\end{array}$ & $\begin{array}{l}0.50^{\mathrm{a}} \\
1.28^{\mathrm{b}}\end{array}$ \\
\hline
\end{tabular}

Cont., continuous variable. ${ }^{a, b}$ Denote variable measured in plantation estates and smallholdings, respectively. All measurements in the former were averaged across different stand ages.

predictor variables were transformed (logarithm and square-root) prior to analysis. We fitted site (55 levels) as a random effect (Bolker et al. 2009).

To test our third prediction on the effects of environmental features on birds grouped into different foraging guilds, we modelled bird abundance within guilds as a function of managementlevel, stand-level and landscape-level attributes using GLMMs. We modelled seven common types of foraging guild using GLMMs with site as a random effect, and conducted model selection as described above.

\section{RESULTS}

We recorded 15540 individual birds of 208 species, $32 \%$ of the 648 species known in Peninsular
Malaysia (Jeyarajasingam \& Pearson 1999), including 178 resident species. In logged peat swamp forest, plantation estates and smallholdings we recorded 194, 108 and 55 bird species, respectively, including forest-dependent species, open-area species and wetland species. There was overlap in the occurrence of bird species of logged peat swamp forest, plantation estates and smallholdings. Fifty species occurred in all three broad groups of sites.

\section{Diversity of foraging guilds}

As expected, logged peat swamp forest supported more guilds (mean $=12.3$, or $65 \%$ of total foraging guilds) than oil palm plantation estates $($ mean $=6.2)$ and smallholdings $\quad($ mean $=6.6)$ (Wald statistic $=45.71, \quad P<0.001)$. Few forest 
bird species were recorded in oil palm landscapes, but generalists were abundant (Fig. 1). Most foraging guilds (except for the diurnal raptors) were dominated by single species. For example, the Oriental Magpie-robin Copsychus saularis and Red Junglefowl Gallus gallus were, respectively, the most common terrestrial gleaning insectivores and terrestrial omnivores.

At least 10 insectivorous species were recorded in the terrestrial gleaning guild in logged peat swamp forest, whereas in oil palm landscapes, this guild was represented by very few species (e.g. Oriental Magpie-robin and Common Tailorbird Orthotomus sutorius). Few arboreal frugivores were recorded in oil palm landscapes (e.g. Hill Myna Gracula religiosa and Rhinoceros Hornbill Buceros rhinoceros), whereas more species from that guild were recorded in logged peat swamp forest.

\section{Factors influencing bird foraging guild diversity in oil palm landscapes}

Results from Model 1 (fitting management regime) showed that increased vegetation cover $(P<0.001)$, reduced canopy cover $(P<0.001)$ and reduced epiphyte persistence $(P<0.035)$ were associated with a higher number of foraging guilds in oil palm landscapes (Table 2; Fig. 2). Management regime had

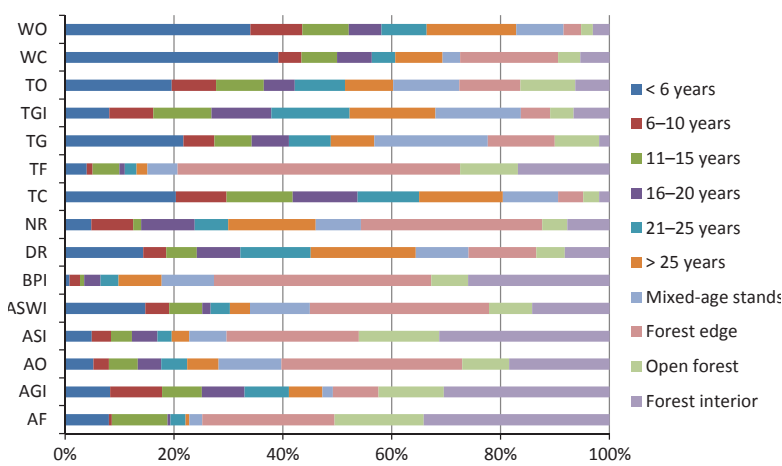

Figure 1. Foraging guilds of insectivorous birds, non-insectivorous birds, and raptor and wetland species observed in oil palm landscapes and forest habitats. Bar charts are based on relative abundance for different foraging guilds. The 15 most common guilds are coded as followed: TG, terrestrial granivore; TF, terrestrial frugivore; AF, arboreal frugivore; WO, wetland omnivore; $\mathrm{TO}$, terrestrial omnivore; $\mathrm{AO}$, arboreal omnivore; WC, wetland carnivore; TC, terrestrial carnivore; $\mathrm{NR}$, nocturnal raptor; DR, diurnal raptor; TGI, terrestrial gleaning insectivore; BPI, bark-probing insectivore; ASWI, aerial sweeping insectivore; ASI, arboreal sallying insectivore; AGI, arboreal gleaning insectivore. little influence $(P=0.072)$. Smallholdings and plantation estates supported 11.81 and 10.28 guild types, respectively. The presence or absence of cattle grazing had no significant effect on the number of bird foraging guilds $(P=0.285)$. At the landscape level, none of the predictor variables significantly influenced the number of foraging guilds.

Model 2 (fitted with stand age) suggested that stand age $(P<0.001)$, increased vegetation cover $(P=0.005)$ and reduced canopy cover $(P<0.001)$ had a significant positive influence on the total number of foraging guilds (Table 2). Oil palm stands aged $<6,6-10,11-15,16-20,21-25,>25$ and mixed-age years supported an average of 10.26, $9.51,10.06,9.99,10.63,11.35$ and 11.51 guilds, respectively. Cattle grazing and predation did not significantly influence the number of bird foraging guilds $(P=0.298$ and $P=0.517$, respectively). As in Model 1, none of the predictor variables for landscape level significantly influenced bird foraging guild diversity.

\section{Varying responses of foraging guilds}

Different foraging guilds exhibited different sensitivity to stand-level and landscape-level attributes (Table 3). Most foraging guilds responded only to stand-level attributes and not to landscape-level attributes (Figs 3-5). Birds from six different guilds responded to the type of oil palm management regime: arboreal gleaning insectivores, arboreal omnivores, arboreal sweeping insectivores, terrestrial gleaning insectivores, terrestrial frugivores

Table 2. Factors significantly influencing the diversity of bird foraging guilds in 470 transects located in 41 oil palm plantation estates and 14 smallholdings, modelled as a function of stand and landscape-level attributes.

\begin{tabular}{|c|c|c|c|c|}
\hline $\begin{array}{l}\text { Predictor } \\
\text { variable }\end{array}$ & $\begin{array}{l}\text { Parameter } \\
\text { estimate }\end{array}$ & se & $\begin{array}{l}\text { Wald } \\
\text { statistic }\end{array}$ & $P$ \\
\hline \multicolumn{5}{|c|}{ Model 1 - Management regime } \\
\hline Vegetation cover & 0.0016 & 0.0008 & 35.73 & $<0.001$ \\
\hline Canopy cover & -0.0021 & 0.0007 & 43.45 & $<0.001$ \\
\hline $\begin{array}{l}\text { Epiphyte } \\
\text { persistence }\end{array}$ & -0.0012 & 0.0005 & 4.48 & 0.035 \\
\hline Survey time & & & 32.92 & $<0.001$ \\
\hline \multicolumn{5}{|l|}{ Model 2 - Stand age } \\
\hline Stand age & & & 71.37 & $<0.001$ \\
\hline Vegetation cover & 0.0012 & 0.0008 & 7.85 & 0.005 \\
\hline Canopy cover & -0.0025 & 0.0008 & 19.86 & $<0.001$ \\
\hline Survey time & & & 30.57 & $<0.001$ \\
\hline
\end{tabular}



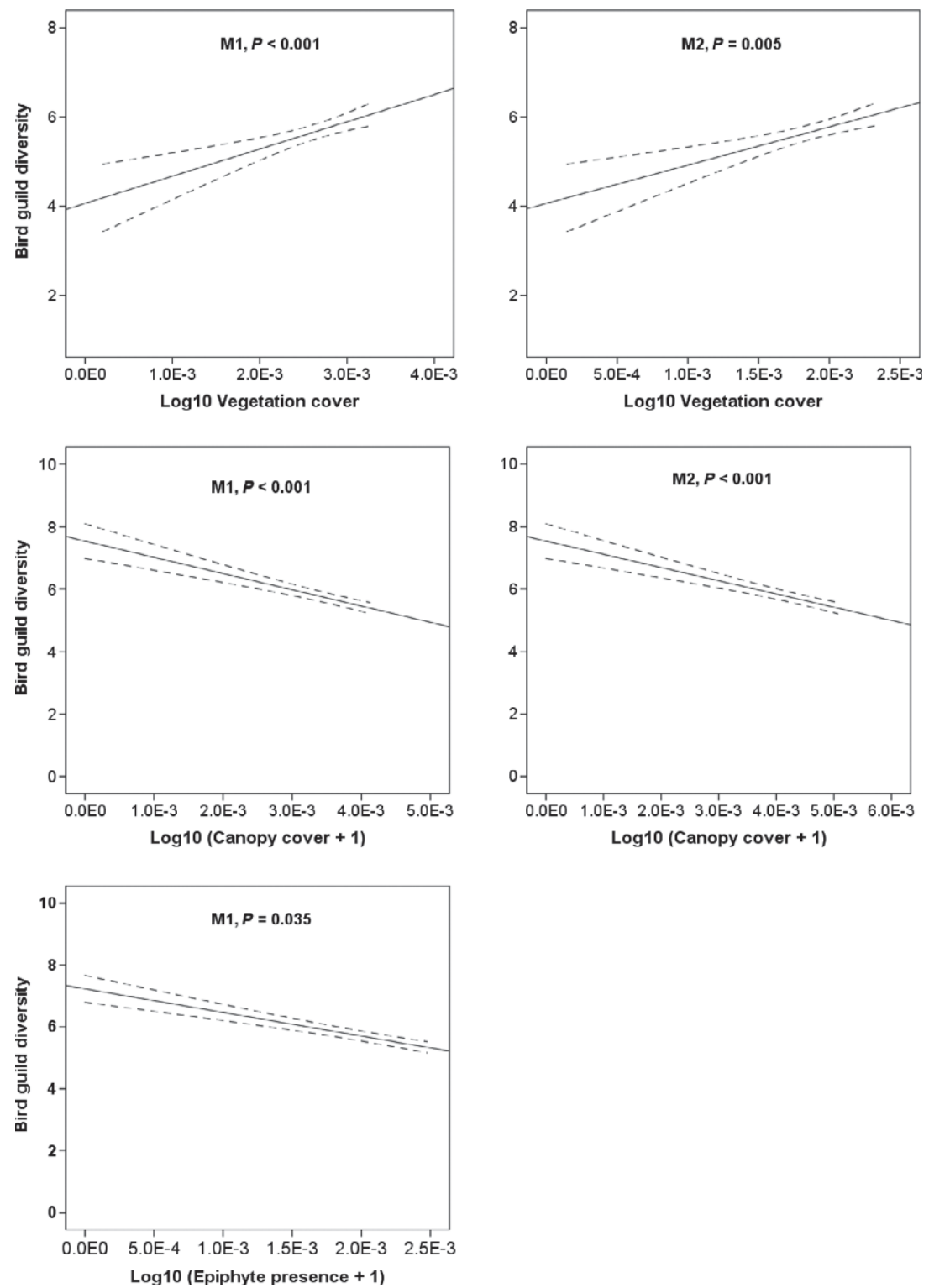

Figure 2. Relationships between bird foraging guild diversity and vegetation cover (top), canopy cover (middle) and epiphyte persistence (lower). Scatter plots have 95\% confidence intervals (dashed) on the regression (solid) line. M1 and M2 denote Model 1 and Model 2, respectively.

and wetland omnivores. Arboreal omnivores (Model 1: $P=0.033$; Model 2: $P=0.003$ ) and terrestrial frugivores (Model 2: $P=0.042$ ) increased in abundance with decreasing distance to the nearest forest patch. The abundance of wetland omnivores decreased significantly with increasing forest cover within $5 \mathrm{~km}$ of each oil palm site (Model 1: $P=0.018$; Model 2: $P=0.012$ ) but increased with increasing isolation from continuous forests (Model
1: $P=0.006$; Model 2: $P=0.049)$. Of the 10 foraging guilds examined, only the arboreal sallying insectivores did not respond to any environmental attributes.

\section{DISCUSSION}

We identified three key patterns in bird functional diversity in logged peat swamp forests, oil 
Table 3. GLMMs of individual responses of nine common bird foraging guilds to various stand and landscape-level attributes.

\begin{tabular}{|c|c|c|c|c|}
\hline Predictor variable & $\begin{array}{c}\text { Parameter } \\
\text { estimate }\end{array}$ & se & $\begin{array}{l}\text { Wald } \\
\text { statistic }\end{array}$ & $P$ \\
\hline \multicolumn{5}{|l|}{ Arboreal gleaning insectivore } \\
\hline \multicolumn{5}{|l|}{ Model 1 - Management regime } \\
\hline Management regime ${ }^{\mathrm{a}}$ & & & 10.60 & 0.001 \\
\hline Canopy cover & 0.0126 & 0.0043 & 7.51 & 0.006 \\
\hline \multicolumn{5}{|l|}{ Model 2 - Stand age } \\
\hline Stand age & & & 23.62 & $<0.001$ \\
\hline Canopy cover & 0.0132 & 0.0049 & 5.51 & 0.019 \\
\hline Survey time & & & 4.25 & 0.040 \\
\hline \multicolumn{5}{|l|}{ Arboreal omnivore } \\
\hline \multicolumn{5}{|l|}{ Model 1 - Management regime } \\
\hline Management regime ${ }^{\mathrm{b}}$ & & & 10.30 & 0.002 \\
\hline Vegetation cover & 0.0072 & 0.0035 & 5.74 & 0.017 \\
\hline Distance to the nearest forest patch & -0.1395 & 0.0680 & 4.61 & 0.033 \\
\hline Survey time & & & 13.28 & $<0.001$ \\
\hline \multicolumn{5}{|l|}{ Model 2 - Stand age } \\
\hline Stand age & & & 29.14 & $<0.001$ \\
\hline Undergrowth height & -0.0060 & 0.0026 & 5.32 & 0.022 \\
\hline Canopy cover & -0.0075 & 0.0036 & 5.12 & 0.024 \\
\hline Distance to the nearest forest patch & -0.1917 & 0.0676 & 8.81 & 0.003 \\
\hline Survey time & & & 11.16 & $<0.001$ \\
\hline \multicolumn{5}{|l|}{ Arboreal sweeping insectivore } \\
\hline \multicolumn{5}{|l|}{ Model 1 - Management regime } \\
\hline Management regime ${ }^{\mathrm{b}}$ & & & 4.35 & 0.041 \\
\hline Vegetation cover & 0.0001 & 0.0050 & 8.69 & 0.003 \\
\hline Canopy cover & -0.0124 & 0.0042 & 27.77 & $<0.001$ \\
\hline \multicolumn{5}{|l|}{ Model 2 - Stand age } \\
\hline Stand age & & & 56.07 & $<0.001$ \\
\hline \multicolumn{5}{|l|}{ Diurnal raptor } \\
\hline \multicolumn{5}{|l|}{ Model 1 - Management regime } \\
\hline Vegetation cover & 0.0182 & 0.0047 & 25.90 & $<0.001$ \\
\hline \multicolumn{5}{|l|}{ Model 2} \\
\hline Stand age & & & 32.59 & $<0.001$ \\
\hline Vegetation cover & 0.0147 & 0.0047 & 12.23 & $<0.001$ \\
\hline \multicolumn{5}{|l|}{ Terrestrial carnivore } \\
\hline \multicolumn{5}{|l|}{ Model 1 - Management regime } \\
\hline Elevation & -0.0162 & 0.0061 & 6.41 & 0.012 \\
\hline Vegetation cover & -0.0001 & 0.0025 & 9.87 & 0.002 \\
\hline Canopy cover & -0.0070 & 0.0021 & 20.69 & $<0.001$ \\
\hline Cattle grazing ${ }^{c}$ & & & 17.71 & $<0.001$ \\
\hline \multicolumn{5}{|l|}{ Model 2 - Stand age } \\
\hline Stand age & & & 27.41 & $<0.001$ \\
\hline Elevation & -0.0178 & 0.0063 & 10.22 & 0.002 \\
\hline Canopy cover & -0.0061 & 0.0025 & 5.63 & 0.018 \\
\hline Cattle grazing $^{c}$ & & & 17.65 & $<0.001$ \\
\hline \multicolumn{5}{|l|}{ Terrestrial frugivore } \\
\hline \multicolumn{5}{|l|}{ Model 1 - Management regime } \\
\hline Management regime ${ }^{\mathrm{b}}$ & & & 12.33 & $<0.001$ \\
\hline Vegetation cover & -0.0018 & 0.0025 & 4.88 & 0.028 \\
\hline Canopy cover & -0.0067 & 0.0022 & 22.62 & $<0.001$ \\
\hline Cattle grazing $^{c}$ & & & 5.05 & 0.025 \\
\hline Survey time & & & 19.40 & $<0.001$ \\
\hline \multicolumn{5}{|l|}{ Model 2 - Stand age } \\
\hline Stand age & & & 28.68 & $<0.001$ \\
\hline Canopy cover & -0.0086 & 0.0026 & 16.03 & $<0.001$ \\
\hline
\end{tabular}


Table 3. (continued)

\begin{tabular}{|c|c|c|c|c|}
\hline Predictor variable & $\begin{array}{l}\text { Parameter } \\
\text { estimate }\end{array}$ & se & $\begin{array}{l}\text { Wald } \\
\text { statistic }\end{array}$ & $P$ \\
\hline Cattle grazing ${ }^{\mathrm{C}}$ & & & 4.12 & 0.043 \\
\hline Distance to the nearest forest patch & -0.0934 & 0.0485 & 4.18 & 0.042 \\
\hline Survey time & & & 18.49 & $<0.001$ \\
\hline \multicolumn{5}{|l|}{ Terrestrial gleaning insectivore } \\
\hline \multicolumn{5}{|l|}{ Model 1 - Management regime } \\
\hline Management regime ${ }^{\mathrm{b}}$ & & & 4.07 & 0.047 \\
\hline Elevation & 0.0051 & 0.0033 & 6.46 & 0.012 \\
\hline Vegetation cover & 0.0031 & 0.0016 & 13.28 & $<0.001$ \\
\hline Undergrowth height & 0.0042 & 0.0010 & 21.97 & $<0.001$ \\
\hline Canopy cover & 0.0037 & 0.0014 & 4.82 & 0.029 \\
\hline Survey time & & & 45.90 & $<0.001$ \\
\hline \multicolumn{5}{|l|}{ Model 2 - Stand age } \\
\hline Stand age & & & 158.18 & $<0.001$ \\
\hline Elevation & 0.0067 & 0.0031 & 10.29 & 0.002 \\
\hline Vegetation cover & 0.0012 & 0.0015 & 7.25 & 0.007 \\
\hline Undergrowth height & 0.0022 & 0.0009 & 5.68 & 0.018 \\
\hline Canopy cover & -0.0024 & 0.0015 & 5.02 & 0.026 \\
\hline Survey time & & & 51.13 & $<0.001$ \\
\hline \multicolumn{5}{|l|}{ Terrestrial omnivore } \\
\hline \multicolumn{5}{|l|}{ Model 1 - Management regime } \\
\hline Vegetation cover & 0.0027 & 0.0020 & 81.00 & $<0.001$ \\
\hline Undergrowth height & 0.0059 & 0.0012 & 11.54 & $<0.001$ \\
\hline Canopy cover & -0.0073 & 0.0017 & 62.66 & $<0.001$ \\
\hline Predation & -0.0238 & 0.0118 & 4.91 & 0.027 \\
\hline Survey time & & & 17.15 & $<0.001$ \\
\hline \multicolumn{5}{|l|}{ Model 2 - Stand age } \\
\hline Stand age & & & 90.57 & $<0.001$ \\
\hline Vegetation cover & 0.0031 & 0.0020 & 38.48 & $<0.001$ \\
\hline Undergrowth height & 0.0063 & 0.0012 & 23.59 & $<0.001$ \\
\hline Canopy cover & -0.0060 & 0.0019 & 16.98 & $<0.001$ \\
\hline Predation & -0.0216 & 0.0118 & 4.28 & 0.039 \\
\hline Survey time & & & 17.40 & $<0.001$ \\
\hline \multicolumn{5}{|l|}{ Wetland omnivore } \\
\hline \multicolumn{5}{|l|}{ Model 1 - Management regime } \\
\hline Management regime ${ }^{\mathrm{b}}$ & & & 4.92 & 0.027 \\
\hline Vegetation cover & 0.0043 & 0.0043 & 51.26 & $<0.001$ \\
\hline Canopy cover & -0.0094 & 0.0033 & 50.76 & $<0.001$ \\
\hline Epiphyte persistence & -0.0072 & 0.0025 & 6.96 & 0.009 \\
\hline Forest cover & $-2.8 \times 10^{-5}$ & $-1.2 \times 10^{-5}$ & 5.61 & 0.018 \\
\hline Isolation from continuous forest & 0.0260 & 0.0090 & 7.69 & 0.006 \\
\hline Survey time & & & 4.06 & 0.044 \\
\hline \multicolumn{5}{|l|}{ Model 2 - Stand age } \\
\hline Stand age & & & 84.75 & $<0.001$ \\
\hline Vegetation cover & 0.0036 & 0.0044 & 13.71 & $<0.001$ \\
\hline Canopy cover & -0.0099 & 0.0041 & 13.99 & $<0.001$ \\
\hline Forest cover & $-2.9 \times 10^{-5}$ & $-1.1 \times 10^{-5}$ & 6.33 & 0.012 \\
\hline Isolation from continuous forest & 0.0234 & 0.0108 & 4.47 & 0.049 \\
\hline Survey time & & & 4.92 & 0.027 \\
\hline
\end{tabular}

${ }^{\mathrm{a}}$ Higher abundance in plantation estates than smallholdings. ${ }^{\mathrm{b}}$ Higher abundance in smallholdings than plantation estates. ${ }^{\mathrm{c}} \mathrm{Higher}$ abundance with presence of cattle grazing.

palm plantation estates and smallholdings. First, oil palm landscapes, regardless of management regime, supported fewer foraging guilds than did logged peat swamp forest. Secondly, stand structural complexity strongly influenced bird functional diversity in oil palm landscapes. Thirdly, different guilds responded differently to environmental attributes. 

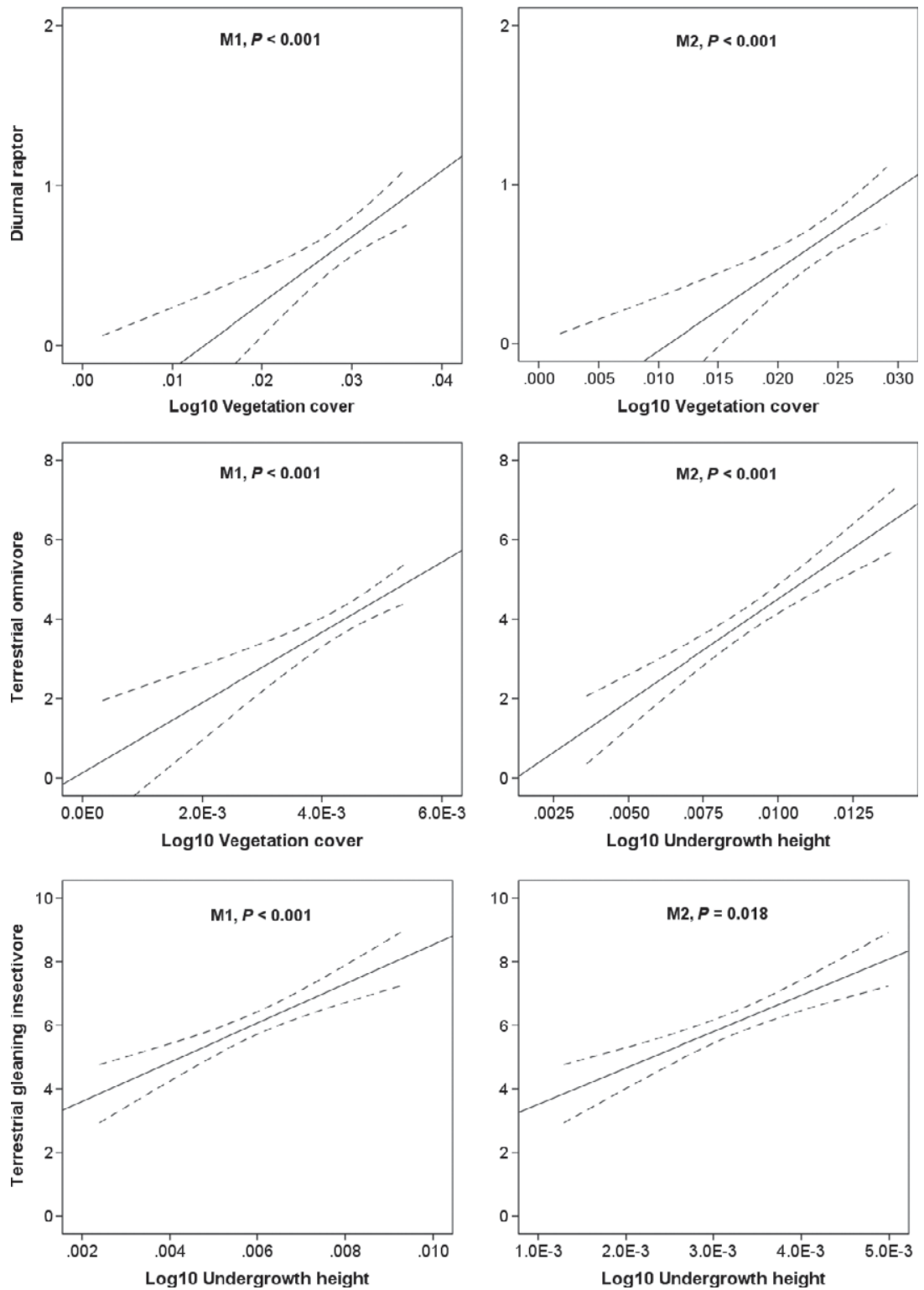

Figure 3. Scatterplots with $95 \%$ confidence intervals (dashed) on the regression (solid) line showing the relationships between the abundance of different foraging guilds and vegetation cover and undergrowth height. M1 and M2 denote Model 1 and Model 2 , respectively.

\section{Diversity of foraging guilds}

Foraging guilds were more diverse in logged peat swamp forest than in oil palm landscapes, perhaps due to greater habitat complexity in logged peat swamp forest. In contrast to oil palm monocultures, the diversity of tree species in logged peat swamp forest is likely to provide habitats and food resources for more bird species. However, omnivorous birds such as Black-naped Oriole Oriolus chinensis, Red Junglefowl and Olive-backed Sunbird Cinnyris jugularis were more likely to occur in oil palm landscapes than in forested areas. Omnivorous birds may rely on ground-layer vegetation despite fewer native trees being available in oil palm landscapes, particularly plantation estates. For example, Red Junglefowl forages on a diverse range of food from plant material (e.g. seeds) to arthropods (e.g. earthworms and termites) (Arshad et al. 2000). In our study sites, both migratory 

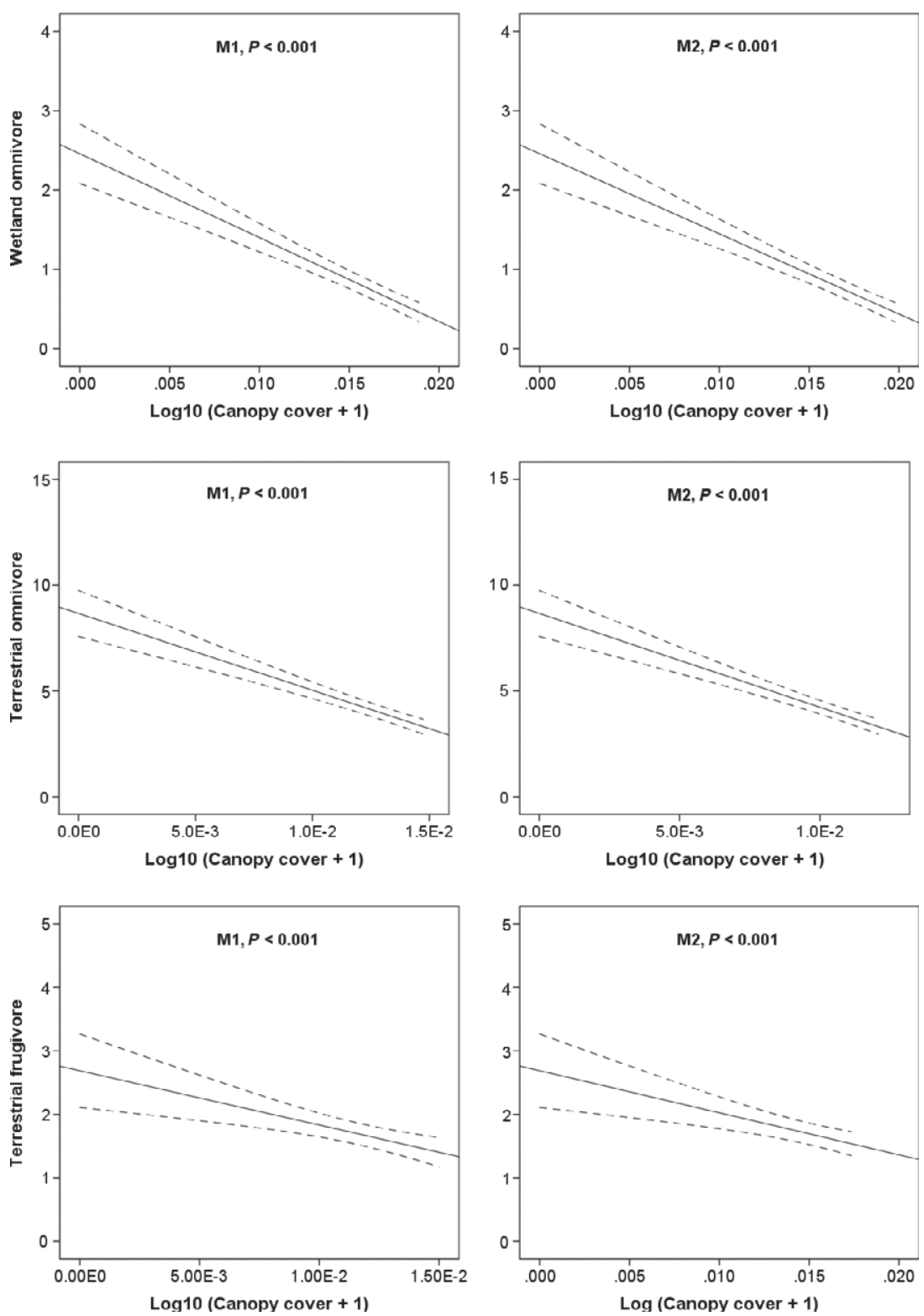

Figure 4. Scatterplots with $95 \%$ confidence intervals (dashed) on the regression (solid) line showing the relationships between the abundance of different foraging guilds and canopy cover. M1 and M2 denote Model 1 and Model 2, respectively.

(e.g. Brown Shrike Lanius cristatus) and resident species (e.g. Common Tailorbird) used undergrowth vegetation. Ground-layer vegetation growing naturally in oil palm landscapes also may provide seeds for granivorous birds. The importance of ground-layer vegetation to birds in oil palm stands also has been noted by Aratrakorn et al. (2006) and Najera and Simonetti (2010b).

Insectivorous birds and diurnal raptors dominated oil palm landscapes in terms of relative abundance. These findings can be attributed to the high biomass of some arthropods, rodents and snakes in plantation landscapes and nearby forest areas (Wong 1986, Turner \& Foster 2009). Consistent with previous studies (Peh et al. 2006, Sheldon et al. 2010), we found that bark-probing insectivores (e.g. woodpeckers) were most abundant in logged peat swamp forest.

Wetland omnivorous and carnivorous birds were more abundant in oil palm landscapes than 

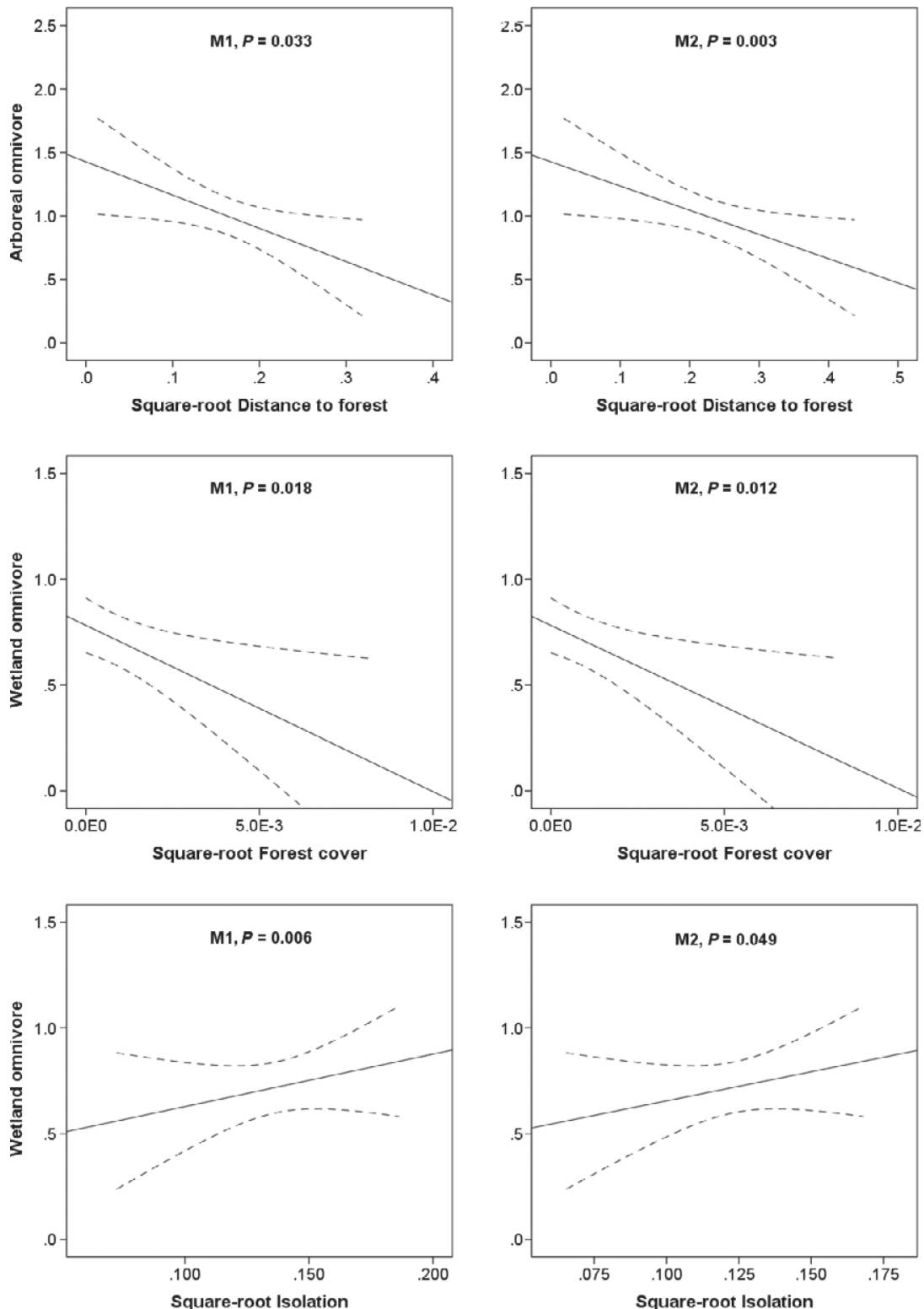

Figure 5. Scatterplots with 95\% confidence intervals (dashed) on the regression (solid) line showing the relationships between the abundance of different foraging guilds and distance to nearest forest patches, forest cover and isolation from continuous forests. M1 and M2 denote Model 1 and Model 2, respectively.

in logged peat swamp forest, due to the ponds and flood-controlled ditches that were common in plantation estates, and which attracted wetland species such as Lesser Whistling-duck Dendrocygna javanica. Such aquatic habitats were better maintained in plantation estates than smallholdings, and offered foraging areas for the White-breasted Waterhen Amaurornis phoenicurus, Purple Heron Ardea purpurea, Yellow Bittern Ixobrychus sinensis and Chinese Pond Heron Ardeola bacchus. In contrast to studies in rubber plantations (Yorke 1984, Beukema et al. 2007), the occurrence of different foraging waterbirds in oil palm landscapes suggests that aquatic habitats in these areas contribute to biodiversity conservation. In contrast, rubber plantations have no similar man-made aquatic habitats and few waterbirds are reported from such plantations (Yorke 1984). 
Terrestrial and arboreal frugivorous birds were more abundant in peat swamp forest than in oil palm landscapes (Fig. 1). A lack of fruit trees and flowering plants probably explained the paucity of frugivorous birds in oil palm cultivation areas. However, large species such as Long-tailed Parakeet Psittacula longicauda and Oriental Pied Hornbill Anthracoceros albirostris forage in plantation estates, probably due to proximity to areas of natural forest (Luck \& Daily 2003, Anand et al. 2008). Agronomists have reported the consumption of oil palm fruits by Black Vulture Coragyps atratus in Brazil (Piggott 1990) and Long-tailed Parakeet $P$. longicauda and Blue-rumped Parrot Psittinus cyanurus in Malaysia (Turner \& Gillbanks 1974).

\section{Factors related to foraging guild diversity in oil palm}

The stand age of oil palm was an important determinant of foraging guild diversity, probably because it is a proxy for a wide range of environmental attributes, including microclimate and vegetation structure. For example, we detected fewer bark-probing woodpeckers such as Common Flameback Dinopium javanense and Rufous Woodpecker Micropternus brachyurus in young oil palm stands. These birds were more often found in older stands (especially those over 16 years old), possibly because they contain more abundant suitable food (Bruhl \& Eltz 2010, Fayle et al. 2010). The presence of dead or old oil palm plants may explain the detection of this guild in plantation estates as well as in smallholdings.

The foraging guild diversity in oil palm was inversely related to canopy cover. This can be attributed to the availability of light penetrating to the ground layer, with more sunlight encouraging ground-layer vegetation growth, and in turn benefitting foraging birds (Sheldon et al. 2010). In addition, arthropods such as dragonflies, which are consumed by insectivorous birds (e.g. Blue-throated Bee-eater Merops viridis), are active in open areas exposed to direct sunlight.

Smallholdings supported a higher foraging guild diversity than plantation estates, with more forest species recorded in smallholdings than in plantation estates. Those species in smallholdings included Rhinoceros Hornbill, Oriental Pied Hornbill, Hill Myna, Crested Serpent-eagle Spilornis cheela, Longtailed Parakeet and Asian Paradise-flycatcher
Terpsiphone paradisi. The occurrence of forest species may be attributed to habitat complexity in oil palm smallholdings. Plant diversity and multi-strata canopy characterize smallholdings but are rare in most plantation estates.

There was no relationship between landscapelevel attributes (e.g. distance to nearest natural forest patches, forest cover of primary or secondary forest within $5 \mathrm{~km}$, and isolation from continuous forests) and the diversity of bird foraging guilds in oil palm landscapes. These results may be attributed to the low dependence of the majority of foraging birds in oil palm areas on forest habitats and resources. Unlike forest species, birds in oil palm areas probably find food resources (e.g. arthropods and seeds) that are readily and locally available in oil palm landscapes. In addition, seed-eaters may benefit from the abundant supply of wild grasses on harvesting paths. Other studies have shown ground-layer vegetation cover and undergrowth height attracted birds by providing seed and invertebrate food sources (Atkinson et al. 2005, Clough et al. 2007).

One caveat to our interpretations results from our lack of data on current cattle stocking densities in oil palm landscapes. Most plantation estates do not permit cattle. However, some allow cattle owned by workers or local people to graze within estates. Unfortunately, no data were available and therefore our analyses were confined to information on the presence/absence of cattle grazing rather than their abundance.

\section{Individualistic foraging guild responses}

Birds grouped into different foraging guilds displayed different responses to environmental conditions, so the occurrence of a particular foraging guild may not be a good indicator of overall bird functional diversity (Lindenmayer \& Franklin 2002). For example, the occurrence of forest species such as the Rhinoceros Hornbill and Oriental Pied Hornbill in oil palm landscapes may not reflect the occurrence of other foraging birds, particularly guilds that rely on specific forest trees or microclimates.

Some foraging birds responded to a particular management regime of oil palm cultivation. For example, arboreal frugivores occurred at a lower abundance in smallholdings than in plantation estates, despite the presence of fruit trees in smallholdings. However, terrestrial frugivores were the 
largest group in smallholdings. These idiosyncratic, guild-level responses to environmental conditions are similar to the responses of birds at the species level (Manning et al. 2004, Barlow et al. 2007).

\section{Conservation implications}

To improve the conservation value of oil palm landscapes for birds, our results suggest four measures. First, maintaining ground-layer vegetation could increase the diversity of bird guilds and to benefit individual foraging groups. Secondly, birds may benefit from canopy pruning to permit light penetration to the ground and encourage the growth of understorey vegetation. Birds may find food sources such as seeds and invertebrates provided by such understorey vegetation. Thirdly, establishment of indigenous fruit trees such as Ficus spp. could benefit large frugivorous birds such as hornbills (Lambert 1991, Lambert \& Marshall 1991). Fourthly, promote the retention of natural and/or secondary forest patches within and surrounding oil palm areas (Koh 2008), whereby the value of these patches depends on the size and isolation from contiguous forests. Apart from providing refuge for various bird species, forest patches may facilitate the movement of forest birds through oil palm landscapes.

The individual responses of each foraging guild indicated that biodiversity conservation in oil palm landscapes demands multiple measures that are implemented in both plantation estates and smallholdings. Hence, palm oil stakeholders should incorporate these measures into existing sustainable palm oil certification schemes (e.g. Roundtable on Sustainable Palm Oil's Principles and Criteria) to produce more biodiversity-friendly palm oil products (Groom et al. 2008, Laurance et al. 2010).

The conservation value of oil palm areas should not be determined solely by the occurrence of rare or endangered species. Although none of the species we recorded in oil palm areas is at present of high conservation concern, these birds may play important ecological roles in oil palm landscapes and nearby natural forest habitats. New bird extinctions in oil palm landscapes would reduce functional diversity even further after forest conversion (Edwards et al. 2013). Therefore, oil palm management regimes (i.e. plantation estates and smallholdings) need to be managed for conservation outcomes as well as for palm oil yield. In addition, most protected reserves in Malaysia are surrounded by large areas of oil palm. Reserves are likely to be more effective if they are surrounded by biodiversity-friendly plantations than intensively managed plantations.

This study was supported by the Ministry of Higher Education Malaysia through the Fundamental Research Grant Scheme, the Wildlife Conservation Society's Research Fellowship Grant 2009, Malaysia Nature Society's Tan Kean Chong Bird Conservation Memorial Fund, and The Australian National University's Field Trip Grant 2009. We are grateful to a number of people in Australia and Malaysia for assistance with various aspects of this study, namely J. Stein, K. Stagoll, A. Jambari, N. L. Ibrahim and Y. Zakaria of the Selangor Forestry Department, and R. Topani of the Department of Wildlife and National Park. A. Jambari and N. L. Ibrahim were employed in the fieldwork. We also thank all plantation managers and staff from Sime Darby Plantation, United Plantation, Malaysia Airport's Agriculture and Horticulture, FELDA, FELCRA and Perbadanan Kemajuan Pertanian Selangor. We thank S. Marsden and two anonymous referees for their constructive comments and suggestions, which greatly improved our manuscript.

\section{REFERENCES}

Abrahamczyk, S., Kessler, M., Putra, D.D., Waltert, M. \& Tscharntke, T. 2008. The value of differently managed cacao plantations for forest bird conservation in Sulawesi, Indonesia. Bird Conserv. Int. 18: 349-362.

Anand, M.O., Krishnaswamy, J. \& Das, A. 2008. Proximity to forests drives bird conservation value of coffee plantations: implications for certification. Ecol. Appl. 18: 1754-1763.

Anderson, D.R., Laake, J.L., Crain, B.R. \& Burnham, K.P. 1979. Guidelines for line transect sampling of biological populations. J. Wildl. Manage. 43: 70-78.

Aratrakorn, S., Thunhikorn, S. \& Donald, P.F. 2006. Changes in bird communities following conversion of lowland forest to oil palm and rubber plantations in southern Thailand. Bird Conserv. Int. 16: 71-82.

Arshad, M.I., Zakaria, M., Sajap, A.S. \& Ismail, A. 2000. Food and feeding habits of Red Junglefowl. Pak. J. Biol. Sci. 3: 1024-1026.

Atkinson, P.W., Fuller, R.J., Vickery, J.A., Conway, G.J., Tallowin, J.R.B., Smith, R.E.N., Haysom, K.A., Ings, T.C., Asteraki, E.J. \& Brown, V.K. 2005. Influence of agricultural management, sward structure and food resources on grassland field use by birds in lowland England. J. Appl. Ecol. 42: 932-942.

Barlow, J., Mestre, L.A.M., Gardner, T.A. \& Peres, C.A. 2007. The value of primary, secondary and plantation forests for Amazonian birds. Biol. Conserv. 136: 212-231.

Beukema, H., Danielsen, F., Vincent, G., Hardiwinoto, S. \& van Andel, J. 2007. Plant and bird diversity in rubber agroforests in the lowlands of Sumatra, Indonesia. Agroforest. Sys. 70: 217-242. 
Blake, J.G. 1983. Trophic structure of bird communities in forest patches in East-Central Illinois. Wilson Bull. 95: 416-430.

Bolker, B.M., Brooks, M.E., Clark, C.J., Geange, S.W., Poulsen, J.R., Stevens, M.H.H. \& White, J.S.S. 2009. Generalized linear mixed models: a practical guide for ecology and evolution. Trends Ecol. Evol. 24: 127-135.

Bruhl, C.A. \& Eltz, T. 2010. Fuelling the biodiversity crisis: species loss of ground-dwelling forest ants in oil palm plantations in Sabah, Malaysia (Borneo). Biodivers. Conserv. 19: 519-529.

Butler, R.A. \& Laurance, W.F. 2009. Is oil palm the next emerging threat to the Amazon? Trop. Conserv. Sci. 2: 1-10.

Carlo, T.A., Collazo, J.A. \& Groom, M.J. 2004. Influences of fruit diversity and abundance on bird use of two shaded coffee plantations. Biotropica 36: 602-614.

Clough, Y., Kruess, A. \& Tscharntke, T. 2007. Local and landscape factors in differently managed arable fields affect the insect herbivore community of a non-crop plant species. J. Appl. Ecol. 44: 22-28.

Danielsen, F., Beukema, H., Burgess, N.D., Parish, F., Bruhl, C.A., Donald, P.F., Murdiyarso, D., Phalan, B., Reijnders, L., Struebig, M. \& Fitzherbert, E.B. 2009. Biofuel plantations on forested lands: double jeopardy for biodiversity and climate. Conserv. Biol. 23: 348-358.

De Chenon, R.D. \& Susanto, A. 2006. Ecological observations on diurnal diurnal birds in Indonesian oil palm plantations. J. Oil Palm R. 2006 (Spec Issue April): 122-143.

Dietsch, T.V., Perfecto, I. \& Greenberg, R. 2007. Avian foraging behavior in two different types of coffee agroecosystem in Chiapas, Mexico. Biotropica 39: 232-240.

Donald, P.F. 2004. Biodiversity impacts of some agricultural commodity production systems. Conserv. Biol. 18: 17-37.

Edwards, D.P., Hodgson, J.A., Hamer, K.C., Mitchell, S.L., Ahmad, A.H., Cornell, S.J. \& Wilcove, D.S. 2010. Wildlifefriendly oil palm plantations fail to protect biodiversity effectively. Conserv. Lett. 3: 236-242.

Edwards, F.A., Edwards, D.P., Hamer, K.C. \& Davies, R.G. 2013. Impacts of logging and conversion of rainforest to oil palm on the functional diversity of birds in Southeast Asia. Ibis 155: 313-326.

Estrada, C.G., Damon, A., Hernandez, C.S., Pinto, L.S. \& Nunez, G.I. 2006. Bat diversity in montane rainforest and shaded coffee under different management regimes in southeastern Chiapas, Mexico. Biol. Conserv. 132: 351-361.

Fayle, T.M., Turner, E.C., Snaddon, J.L., Chey, V.K., Chung, A.Y.C., Eggleton, P. \& Foster, W.A. 2010. Oil palm expansion into rain forest greatly reduces ant biodiversity in canopy, epiphytes and leaf-litter. Basic Appl. Ecol. 11: 337-345.

Felton, A., Wood, J., Felton, A.M., Hennessey, B. \& Lindenmayer, D.B. 2008. Bird community responses to reduced-impact logging in a certified forestry concession in lowland Bolivia. Biol. Conserv. 141: 545-555.

Gardner, T.A., Barlow, J., Parry, L.W. \& Peres, C.A. 2007. Predicting the uncertain future of tropical forest species in a data vacuum. Biotropica 39: 25-30.

Greenberg, R., Bichier, P., Angon, A.C. \& Reitsma, R. 1997. Bird populations in shade and sun coffee plantations in central Guatemala. Conserv. Biol. 11: 448-459.

Groom, M.J., Gray, E.M. \& Townsend, P.A. 2008. Biofuels and biodiversity: principles for creating better policies for biofuel production. Conserv. Biol. 22: 602-609.
Jeyarajasingam, A. \& Pearson, A. 1999. A Field Guide to the Birds of West Malaysia and Singapore. Oxford: Oxford University Press.

Koh, L.P. 2008. Can oil palm plantations be made more hospitable for forest butterflies and birds? J. Appl. Ecol. 45: 1002-1009.

Lambert, F. 1991. The conservation of fig-eating birds in Malaysia. Biol. Conserv. 58: 31-40.

Lambert, F.R. \& Marshall, A.G. 1991. Keystone characteristics of bird-dispersed ficus in a Malaysian lowland rain-forest. J. Ecol. 79: 793-809.

Laurance, W.F., Koh, L.P., Butler, R., Sodhi, N.S., Bradshaw, C.J.A., Neidel, J.D., Consunji, H. \& Vega, J.M. 2010. Improving the performance of the Roundtable on Sustainable Palm Oil for nature conservation. Conserv. Biol. 24: 377-381.

Lindenmayer, D.B. \& Franklin, J.F. 2002. Conserving Forest Biodiversity. Washington, DC: Island Press.

Luck, G.W. \& Daily, G.C. 2003. Tropical countryside bird assemblages: richness, composition, and foraging differ by landscape context. Ecol. Appl. 13: 235-247.

Mackenzie, D.I., Nichols, J.D., Sutton, N., Kawanishi, K. \& Bailey, L.L. 2005. Improving inferences in population studies of rare species that are detected imperfectly. Ecology 86: 1101-1113.

Malaysia Palm Oil Board. 2011. Economic and Statistics. Download from http://www.mpob.gov.my/.

Manning, A.D., Lindenmayer, D.B. \& Nix, H.A. 2004. Continua and umwelt: novel perspectives on viewing landscapes. Oikos 104: 621-628.

Mas, A.H. \& Dietsch, T.V. 2004. Linking shade coffee certification to biodiversity conservation: butterflies and birds in Chiapas, Mexico. Ecol. Appl. 14: 642-654.

Miller, J.R. \& Cale, P. 2000. Behavioral mechanisms and habitat use by birds in a fragmented agricultural landscape. Ecol. Appl. 10: 1732-1748.

Najera, A. \& Simonetti, J.A. 2010a. Can oil palm plantations become bird friendly? Agroforest. Sys. 80: 203-209.

Najera, A. \& Simonetti, J.A. 2010b. Enhancing avifauna in commercial plantations. Conserv. Biol. 24: 319-324.

Peh, K.S.H., Sodhi, N.S., de Jong, J., Sekercioglu, C.H., Yap, C.A.M. \& Lim, S.L.H. 2006. Conservation value of degraded habitats for forest birds in southern Peninsular Malaysia. Divers. Distrib. 12: 572-581.

Piggott, C.J. 1990. Growing Oil Palms: An Illustrated Guide. Kuala Lumpur: The Incorporated Society of Planters.

Reitsma, R., Parrish, J.D. \& McLarney, W. 2001. The role of cacao plantations in maintaining forest avian diversity in southeastern Costa Rica. Agroforest. Sys. 53: 185-193.

Robson, C. 2008. A Guide to the Birds of South-east Asia. London: New Holland.

Schall, R. 1991. Estimation in generalized linear-models with random effects. Biometrika 78: 719-727.

Scharringa, J. 2005. Birds of Tropical Asia 3: Sounds and Sights. Enschede: Bird Songs International.

Sekercioglu, C.H., Ehrlich, P.R., Daily, G.C., Aygen, D., Goehring, D. \& Sandi, R.F. 2002. Disappearance of insectivorous birds from tropical forest fragments. Proc. Natl Acad. Sci. U S A 99: 263-267.

Sekercioglu, C.H., Loarie, S.R., Brenes, F.O., Ehrlich, P.R. \& Daily, G.C. 2007. Persistence of forest birds in the Costa Rican agricultural countryside. Conserv. Biol. 21: 482-494. 
Sheldon, F.H., Styring, A. \& Hosner, P.A. 2010. Bird species richness in a Bornean exotic tree plantation: a long-term perspective. Biol. Conserv. 143: 399-407.

Simberloff, D. \& Dayan, T. 1991. The guild concept and the structure of ecological communities. Ann. Rev. Ecol. Syst. 22: 115-143.

Soderstrom, B., Kiema, S. \& Reid, R.S. 2003. Intensified agricultural land-use and bird conservation in Burkina Faso. Agr. Ecosyst. Environ. 99: 113-124.

Tscharntke, T., Sekercioglu, C.H., Dietsch, T.V., Sodhi, N.S., Hoehn, P. \& Tylianakis, J.M. 2008. Landscape constraints on functional diversity of birds and insects in tropical agroecosystems. Ecology 89: 944-951.

Turner, E.C. \& Foster, W.A. 2009. The impact of forest conversion to oil palm on arthropod abundance and biomass in Sabah, Malaysia. J. Trop. Ecol. 25: 23-30.

Turner, P.D. \& Gillbanks, R.A. 1974. Oil Palm Cultivation and Management. Kuala Lumpur: The Incorporated Society of Planters.

Wilcove, D.S. \& Koh, L.P. 2010. Addressing the threats to biodiversity from oil-palm agriculture. Biodivers. Conserv. 19: 999-1007.
Wong, M. 1986. Trophic organization of understory birds in a Malaysian dipterocarp forest. Auk 103: 100-116.

Yorke, C.D. 1984. Avian community structure in two modified Malaysian habitats. Biol. Conserv. 29: 345-362.

Received 30 March 2011; revision accepted 27 December 2012. Associate Editor: Stuart Marsden.

\section{SUPPORTING INFORMATION}

Additional Supporting Information may be found in the online version of this article:

Table S1. List of 208 bird species and 19 bird guilds recorded in 55 oil palm landscapes and a logged peat swamp forest in Peninsular Malaysia.

Figure S1. Study areas in Perak, Selangor and Negeri Sembilan in Peninsular Malaysia. 